\title{
Totally Thoracoscopic Redo Mitral Valve Replacement for a High-Risk Patient Following Failed MitraClip Procedure
}

\author{
Peijian Wei, MD, ${ }^{1,2}$ Weitao Zhuang, MD, ${ }^{2,3}$ Yanjun Liu, MD, ${ }^{1}$ Jiexu Ma, MD,${ }^{1}$ Wei Zhu, MD, ${ }^{4}$ \\ Jian Zhuang, MD, PhD, ${ }^{1}$ Jian Liu, $\mathrm{MD},{ }^{1}$ Huiming Guo, $\mathrm{MD}, \mathrm{PhD}^{1}$ \\ ${ }^{1}$ Department of Cardiovascular Surgery, Guangdong Cardiovascular Institute, Guangdong Provincial People’s Hospital (Guangdong \\ Academy of Medical Sciences), Guangzhou, China; ${ }^{2}$ Shantou University Medical College, Shantou, China; ${ }^{3}$ Department of Thoracic \\ Surgery, Guangdong Provincial People’s Hospital, Guangdong Academy of Medical Sciences, Guangzhou 510080, China; ${ }^{4}$ Department \\ of Echocardiography, Guangdong Provincial People's Hospital (Guangdong Academy of Medical Sciences), Guangzhou, China
}

\section{ABSTRACT}

The wide adoption of the MitraClip procedure in clinical practice inevitably causes increases in surgical intervention demand for patients following failed MitraClip implantation. Current reports about surgical intervention after failed MitraClip procedure focused on open-heart surgery. In this case, totally thoracoscopic third-time redo mitral valve replacement was successfully performed for a high-risk patient, following aortic valve replacement and a failed MitraClip procedure.

\section{INTRODUCTION}

The MitraClip procedure widely has been performed in a growing number of patients with mitral regurgitation for its reported safety and significant efficacy in elderly and other high-risk patients [Puls 2016]. Meanwhile, an increasing number of patients (2\%-6\%) require surgical intervention within 1 year after MitraClip therapy, due to various complications.

Resternotomy is the mainstream treatment for them. Considering the high-risk profiles of the patients and worsening valvular condition caused by a failed MitraClip, the optimal surgical strategies haven't yet been well established. Is minimally invasive surgery still possible for them? Recently, totally thoracoscopic mitral valve replacement successfully was performed for a high-risk patient, following aortic valve replacement and failed MitraClip procedure in our institution, which provides an answer. It was approved by the ethics committee of Guangdong Provincial People's Hospital (No. KY-Q-2021088-01). Individual informed consent was waived.

Received fuly 8, 2021; accepted fuly 27, 2021.

Correspondence: Hui-Ming Guo and Fian Liu, Department of Cardiovascular Surgery, Guangdong Cardiovascular Institute, Guangdong Provincial People's Hospital (Guangdong Academy of Medical Sciences). No.106, Zhongshan Er Rd, Guangzhou, Guangdong 510080, China (e-mail: guobuiming@gdph.org. cn; 7ameslau1984@sina.com).

\section{CASE REPORT}

A 49-year-old man complaining of shortness of breath on minimal exertion, orthopnea, and other acute left-sided heart failure symptoms for about 10 days was referred to our hospital in September 2020. He reported a medical history of aortic valve replacement in 2001, for severe congenital bicuspid aortic valve stenosis and transcatheter mitral valve repair using two MitraClip devices consecutively in July 2020 for severe mitral regurgitation. Transthoracic echocardiogram (TTE) revealed a preserved ejection fraction, a functional mechanical aortic prosthetic valve, mitral regurgitation grade 4+ due to the two detached MitraClip devices, tricuspid regurgitation grade $2+$, and severe pulmonary hypertension. TTE and enhanced computed tomography images showed that one clip had gripped partly the segment A2 on the anterior leaflet, while the other one had gripped the segment P2 on the posterior leaflet. (Figure 1) After admission, his acute left-sided heart failure symptoms were not improved, despite medication therapy, such as diuretic and vasodilator. Even worse, the patient later developed low cardiac output syndrome, so an intra-aortic balloon pump (IABP) was inserted as an emergency treatment. After discussions in a multidisciplinary heart team, we decided to perform the redo mitral valve surgery via totally thoracoscopic approach instead of traditional sternotomy, considering the poor general situation and extremely high perioperative risk (EuroScore II 36.08\%, STS PROM 42.05\%).

After the achievement of anesthesia, transesophageal echocardiography (TEE) confirmed that the two clips had gripped partly the A2 segment and P2 segment, respectively, which resulted in an extremely severe mitral regurgitation. After systemic heparinization, cardiopulmonary bypass (CPB) was initiated using cannulation through the right femoral artery, right femoral vein, and superior vena cava (cannulation through a right jugular vein by an anesthesiologist), and IABP was suspended subsequently. Then three small ports were made at the right chest wall for intracardiac operation as previously described [Liu 2020]. Dense pericardial adhesions, due to the previous open-heart aortic valve replacement, were observed after the pericardiotomy. After multiple attempts, we found it was impossible to successfully dissect the ascending aorta. So, we decided to perform the totally thoracoscopic mitral valve 


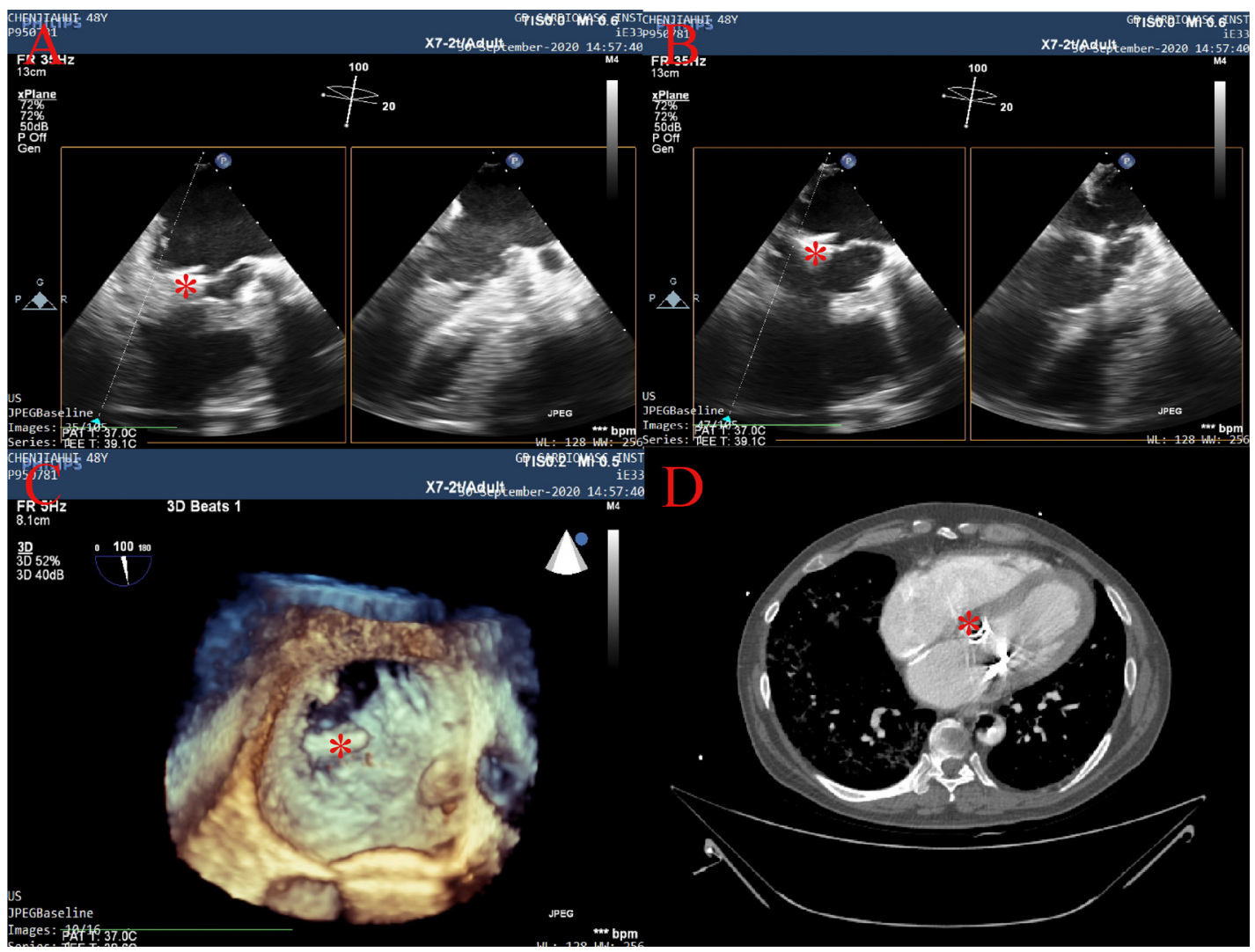

Figure 1. Preprocedural evaluation. (A, B) Transthoracic echocardiogram; (C) Three-dimensional reconstruction form transesophageal echocardiogram; (D) Cardiac computed tomography. The black asterisk indicates the clip on the anterior leaflet of mitral valve.

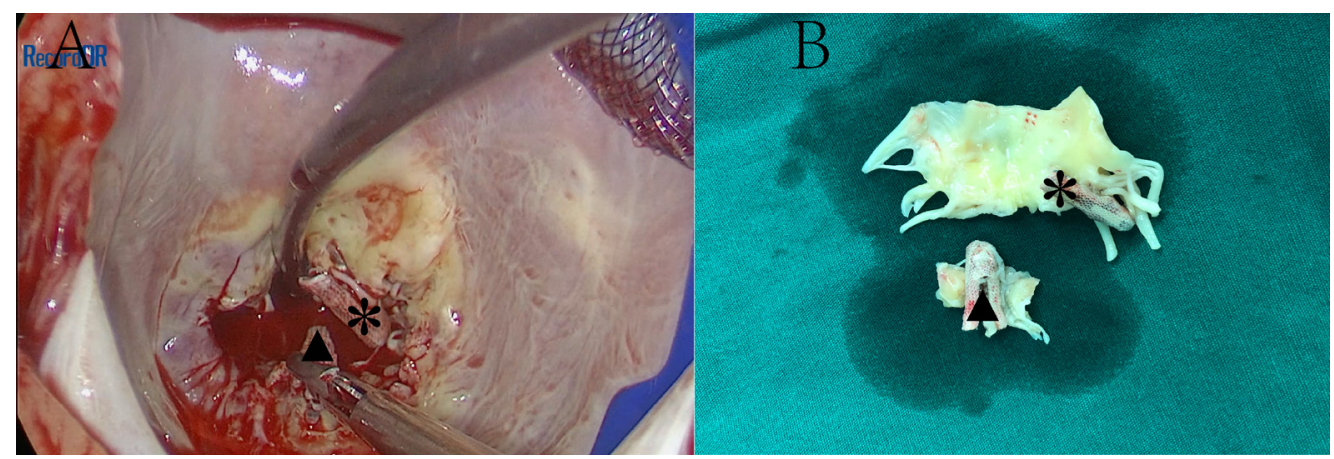

Figure 2. The two clips on the anterior leaflet (the black asterisk) and posterior leaflet (the black triangle) of mitral valve before (A) and after (B) resection.

replacement under ventricular fibrillation without clamping the ascending aorta. A long cannula needle was inserted into the aorta to continuously suck blood for deairing, and the surgical field was constantly flushed with carbon dioxide. Ventricular fibrillation was induced by rapid cooling. Then the left atrium was opened posterior to the interatrial groove, and the mitral valve was well exposed. One clip had gripped only part of the anterior leaflet, and the other one had gripped the posterior leaflet (Figure 2A). (Figure 2) Valve analysis was done using the Carpentier technique. This confirmed type II valvular dysfunction with anterior leaflet prolapse and chordal rupture in the segments A2. We also found that the coaptation height of mitral valve was too short to prevent regurgitation. So, we decided to perform valve replacement instead of repair. The leaflets were excised, and mitral valve replacement routinely was performed using a mechanical prosthetic valve with the consideration of age (Figure 2B). The function of mitral valve prosthesis was confirmed by transesophageal echocardiogram. Then high energy (200 J) asynchronous defibrillation was performed for restoration of sinus rhythm, and the IABP was restarted. One chest tube was placed, and the thoracotomy incision was closed in standard fashion. 
CPB time was 125 minutes. The patient was extubated on postoperative day 4 , and the IABP was weaned off on postoperative day 7 . The patient was discharged from the intensive care unit on postoperative day 9 and discharged home on postoperative day 13. Transthoracic echocardiogram in the first 3 months after operation revealed a good function of the mitral prosthetic valve.

\section{DISCUSSION}

For symptomatic patients with primary severe MR, transcatheter edge-to-edge repair is an alternative to open heart surgery and was outlined as an option for high and prohibitive surgical risk patients in the 2020 ACC/AHA Guideline. However, many patients (2-6\%) require open-heart mitral valve surgery within 1 year for the failed MitraClip implantation [Puls 2016]. The optimal treatment strategy for patients following failed MitraClip procedure remains uncertain, due to the limited data. In a comparative study conducted by Gyoten et al. [Gyoten 2020], in patients with extremely high surgical risk after failed MitraClip therapy, they found that clinical outcomes and survival rate were similar between the repeat MitraClip therapy group and redo surgery group, while medical therapy resulted in poor prognosis. And according to a recent meta-analysis that included 20 reports and consisted of 172 patients by Melillo et al. [Melillo 2020], mitral valve replacement was performed in $68 \%$ of patients after a failed MitraClip procedure. The in-hospital and one-year mortality were $15.0 \%$ and $26.5 \%$, respectively. The poor clinical outcome demonstrated that redo mitral valve surgery after a failed MitraClip procedure represents a great challenge.

In this case, we successfully performed a totally thoracoscopic mitral valve replacement for an extremely high surgical risk patient, following aortic valve replacement and a failed MitraClip procedure. Our case report demonstrated that totally thoracoscopic redo mitral surgery is a potential alternative to open-heart surgery without compromise of quality. It also reminds us that the indications for MitraClip implantation should be discussed carefully beforehand, because the chance of repair after failed intervention drops, and the prognosis after surgery dramatically worsens.

\section{ACKNOWLEDGEMENT}

We would like to thank the patient for making this case possible. Funding: National Key Research and Development Program (2018YFC1002600), Cardiovascular Special Project of Guangdong Provincial People's Hospital (2020XXG010).

\section{REFERENCES}

Gyoten T, Schenk S, Grimmig O, et al. 2020. Outcome of medical therapy, repeat intervention, and mitral valve surgery after failed mitraclip therapy. [published online ahead of print, 2020 Oct 29]. Gen Thorac Cardiovasc Surg. 10.1007/s11748-020-01530-z.

Liu J, Wei P, Ma J, et al. 2020. Propensity-matched analysis of two port approach versus three port approach for totally thoracoscopic mitral valve replacement. J Thorac Dis. 12(10):5986-5995.

Melillo F, Baldetti L, Beneduce A, et al. 2020. Mitral valve surgery after a failed mitraclip procedure. [published online ahead of print, $2020 \mathrm{Nov}$ 22]. Interact Cardiovasc Thorac Surg. ivaa 270

Puls M, Lubos E, Boekstegers P, et al. 2016. One-year outcomes and predictors of mortality after MitraClip therapy in contemporary clinical practice: Results from the german transcatheter mitral valve interventions registry. Eur Heart J. 37(8):703-712. 